Published in final edited form as:

J Urol. 2018 December ; 200(6): 1207-1214. doi:10.1016/j.juro.2018.07.035.

\title{
Impact of the number of cycles of platinum-based first-line chemotherapy for advanced urothelial carcinoma
}

\author{
GP Sonpavde ${ }^{1,{ }^{*}, \text { L Mariani }}{ }^{2, *}$, S Lo Vullo ${ }^{2, *}$, D Raggi ${ }^{2}$, P Giannatempo ${ }^{2}$, A Bamias $^{3}$, SJ \\ Crabb $^{4}$, J Bellmunt ${ }^{5}$, EY Yu ${ }^{6}$, G Niegisch ${ }^{7}$, UN Vaishampayan ${ }^{8}$, C Theodore ${ }^{9}$, DR Berthold ${ }^{10}$, \\ S Srinivas ${ }^{11}$, SS Sridhar ${ }^{12}$, ER Plimack ${ }^{13}$, JE Rosenberg ${ }^{14}$, T Powles $^{15}$, MD Galsky ${ }^{16}$, A \\ Necchi $^{2}$
}

${ }^{1}$ Dana-Farber Cancer Institute, Boston, MA, USA; ${ }^{2}$ Fondazione IRCCS Istituto Nazionale dei Tumori, Milan, Italy; ${ }^{3}$ University of Athens, Greece; ${ }^{4}$ University of Southampton, Southampton, UK; ${ }^{5}$ Hospital del Mar Medical Research Institute (IMIM), Barcelona, Spain; ${ }^{6}$ University of Washington, Seattle, WA, USA; ${ }^{7}$ Heinrich-Heine-University, Medical faculty, Department of Urology, Düsseldorf, Germany; ${ }^{8}$ Wayne State University/Karmanos Cancer Center, Detroit, MI, USA; ${ }^{3}$ Hospital Foch, Suresnes, France; ${ }^{10}$ Centre Hospitalier Universitaire Vaudois, Lausanne, Switzerland; ${ }^{11}$ Stanford University, Stanford, CA, USA; ${ }^{12}$ Princess Margaret Hospital Cancer Center, Toronto, Canada; ${ }^{13}$ Fox Chase Cancer Center, Philadelphia, PA, USA; ${ }^{14}$ Memorial Sloan-

\footnotetext{
Corresponding Author: Andrea Necchi, MD, Department of Medical Oncology, Fondazione IRCCS Istituto Nazionale dei Tumori, Milano, Italy, Tel. 022390 2402; Fax. 022390 3150, andrea.necchi@ istitutotumori.mi.it.

*Equal credit

Publisher's Disclaimer: DISCLAIMER: This is a PDF file of an unedited manuscript that has been accepted for publication. As a service to our subscribers we are providing this early version of the article. The paper will be copy edited and typeset, and proof will be reviewed before it is published in its final form. Please note that during the production process errors may be discovered which could affect the content, and all legal disclaimers that apply to The Journal pertain.
}

Disclosures:

Sonpavde G: Consultant for BMS, Exelixis, Bayer, Sanofi, Pfizer, Novartis, Eisai, Janssen, Amgen, Astrazeneca, Merck Genentech, EMD Serono, Astellas/Agensys; Research support to institution from Bayer, Amgen, Boehringer-Ingelheim, Janssen, Merck, Sanofi, Pfizer; Author for Uptodate; Speaker for Clinical Care Options, Physicians Education Resource (PER), Research to Practice (RTP), Onclive.

- $\quad$ Necchi A: Consulting or Advisory Role: Roche, Bayer, Merck \& Co. Inc., Astra Zeneca, Pfizer, Astellas/Seattle Genetics; Travel, Accommodations, Expenses: Roche, Merck \& Co. Inc., Pierre Fabre, PeerVoice; Research Funding (Institution): Merck \& Co. Inc., Astra Zeneca, Amgen.

- Bellmunt J: Consultant for Bayer, Sanofi, Pfizer, Novartis, Eisai, Janssen, Amgen, Astrazeneca, Merck, Genentech, Agensys, Seattle Genetics; Research support from Novartis and Sanofi; Author for UpTodate.

- $\quad$ Rosenberg JE: consultant to Merck, Bristol-Myers Squibb, Eli Lilly, Agensys, Genentech (Roche), Sanofi, EMD Serono, AstraZeneca, Innovio, Seattle Genetics, Oncogenex, and Bayer; Owns stock in Merck and Illumina.

- $\quad$ Yu EY: Consultant for Astrazeneca, EMD Serono, Incyte, Merck; Research support to institution from Merck, Seattle Genetics.

- $\quad$ Niegisch G: Consultant for Roche Parma AG, IMS Health AG, BMS AG, medac GmbH, MSD Sharp \& Dohme GmbH, Pfizer Pharma; Research support to institution from 4SC AG; lectures: Pfizer Pharma GmbH, Pierre Fabre Pharma GmbH, Roche Pharma AG, MSD Sharp \& Dohme GmbH; travel/accommodation: Roche Parma AG, Pfizer Pharma, BMS AG.

- $\quad$ Galsky MD: Consulting: Astra Zeneca, Bristol-Myers Squibb, Genentech/Roche, Pfizer, Merck \& Co. Inc.

- Mariani L, Lo Vullo S, Raggi D, Giannatempo P, Bamias A, Crabb SJ, Vaishampayan UN, Theodore C, Berthold DR, Srinivas S, Sridhar SS, Plimack ER, Powles T: no relevant disclosures. 
Kettering Cancer Center, New York, NY, USA; ${ }^{15}$ Barts Cancer Institute, Queen Mary University of London, London, United Kingdom; ${ }^{16} \mathrm{Icahn}$ School of Medicine at Mount Sinai, Tisch Cancer Institute, New York, NY, USA

\section{Abstract}

Purpose: To evaluate the impact of number of cycles of platinum-based first-line chemotherapy $<6$ versus the conventional $\prec 6$ on survival of metastatic urothelial carcinoma (UC).

Materials and methods: The Retrospective International Study of Invasive/Advanced Cancer of the Urothelium (RISC) database was employed. The association of the number of cycles of chemotherapy with overall survival (OS) was investigated by Cox multiple regression analysis after controlling for recognized prognostic factors. We excluded patients receiving $<3$ or $>9$ cycles of platinum chemotherapy to reduce confounding factors. The primary analysis was a comparison of OS with 3-5 cycles versus 6-9 cycles using 6-month landmark analysis when 281 death events were observed.

Results: Of 1,020 patients in RISC, 472 who received cisplatin ( $n=338)$ or carboplatin $(n=134)$ were evaluable. A total of 157 patients received 3-5 cycles (median 4) and 315 received 6-9 cycles (median 6). There was no significant difference in OS between 3-5 and 6-9 cycles (HR 1.02, 95\% CI: $0.78-1.33, \mathrm{p}=0.91$ ). No significant interactions were observed with type of platinum $(\mathrm{p}=0.09)$ and completed planned chemotherapy $(\mathrm{p}=0.56)$. Limitations of a hypothesis-generating retrospective analysis apply.

Conclusions: Four cycles of platinum based first-line chemotherapy appear adequate and do not significantly compromise survival of patients with advanced UC. The omission of excessive cycles may avoid unnecessary cumulative toxicities and facilitate better transition to second-line therapy and investigational switch maintenance therapy strategies. These results require prospective validation, but may impact practice in selected patients.

\section{Keywords}

urothelial carcinoma; platinum; first-line chemotherapy; number of cycles; overall survival

\section{Introduction}

Six cycles of platinum-based first-line chemotherapy are conventionally, albeit arbitrarily, targeted in the first-line setting of locally advanced unresectable or metastatic urothelial carcinoma (UC). Cisplatin-eligible patients receive gemcitabine combined with cisplatin (GC) or conventional or dose-dense methotrexate, vinblastine, doxorubicin and cisplatin (MVAC) chemotherapy according to international guidelines ${ }^{1-4}$. A substantial proportion of patients are ineligible for cisplatin due to renal dysfunction, poor performance status or comorbidities, and these patients have historically received carboplatin-based chemotherapy, although the programmed death (PD)-1 and PD-ligand (L)-1 inhibitors, atezolizumab and pembrolizumab, have been approved for this population by both U.S. Food and Drug Administration (FDA) and European Medicines Agency (EMA) ${ }^{5-8}$. Nevertheless, those 
patients receiving first-line therapy with PD-1/PD-L1 inhibitors may still undergo subsequent platinum-based chemotherapy.

However, cisplatin in particular, is associated with significant cumulative toxicities, especially neuropathy, venous thromboembolism and renal dysfunction, which may be irreversible and sometimes lethal, which renders it challenging to deliver the full course of 6 cycles of treatment ${ }^{9,10}$. While carboplatin-based chemotherapy has a more favorable toxicity profile, myelosuppression may lead to infectious complications, thrombocytopenic bleeding and anemia, which may require transfusions. Given that the UC population is frequently elderly and frail, a reduction in number of cycles may confer a more optimal therapeutic index and should be considered if anti-cancer efficacy is not appreciably compromised. Moreover, the choice of 6 cycles of therapy is arbitrary and is not based on prospective comparison with a different number of targeted cycles of therapy. Furthermore, an explosion of new possibilities of active systemic therapies for patients who fail standard first-line chemotherapy has characterized the last few years. Several clinical trials, as well as the use of immune checkpoint inhibitors as standard of care in the second-line setting, may now be offered to patients provided that their performance status is permissive.

Consequently, refining sequencing strategies for advanced UC patients requires special focus on the number of cycles of chemotherapy that should be offered to patients. Since this issue has not been investigated prospectively, we conducted a retrospective analysis.

\section{Patients and Methods}

\section{Study population:}

The Retrospective International Study of Invasive/Advanced Cancer of the Urothelium (RISC) database was employed to conduct a retrospective analysis. RISC is a retrospective study encompassing individual patient-level data from patients with muscle-invasive or advanced UC or non-UC histology who have received systemic therapy during their disease course. This contemporary database includes data gathered from hospitals in the United States, Europe, Israel, and Canada, treated between 2006 and 2011. The RISC study was approved by the ethics committee at each participating institution. In July 2017, data were extracted to select patients with the same characteristics of a previously-published analysis including those with predominant UC histology, cisplatin- or carboplatin-containing combination chemotherapy administered in the first-line metastatic setting, and availability of the number of cycles administered ${ }^{11}$. The data analysis was performed at the Fondazione IRCCS Istituto Nazionale dei Tumori, Milano, Italy. The study was approved by the regulatory committees at each participating institution.

\section{Statistical analyses:}

The study objective was to analyze the association between the total number of cycles of platinum-based combination chemotherapy administered and patient outcome, consisting of the overall survival (OS) as primary endpoint. This association was investigated by a Cox multiple regression analysis controlling for platinum agent (cisplatin or carboplatin) and previously-recognized baseline prognostic factors as reported into the RISC nomogram, i.e. Eastern Cooperative Oncology Group performance status (ECOG-PS), white blood cell 
count, body mass index (BMI), ethnicity, lung, liver, or bone metastases, and prior perioperative chemotherapy ${ }^{11}$. We excluded patients receiving less than 3 or more than 9 cycles of first-line platinum-based chemotherapy to reduce confounding due to early removal for toxicities, early progression or patient decision and increased number of cycles due to response and patient-related factors. The primary analysis was a comparison of 3-5 cycles vs. 6-9 cycles. Supportive sub-analyses were conducted to compare 3-5 vs. 6 vs. 7-9 cycles and exactly 4 vs. exactly 6 cycles, type of platinum (cisplatin or carboplatin) and for those who completed "planned" chemotherapy, according to investigator's reports. Additionally, sensitivity analyses were run excluding patients who had disease progression as best response to treatment. Six-month landmark analysis was applied throughout, accounting for OS events. Statistical analyses were carried out with SAS (version 9. 4, SAS Institute, Cary, NC) and R software (version 3.4.2, R Foundation for Statistical Computing, Vienna, Austria). Statistical significance was set at the conventional 5\% two-sided threshold.

\section{Results}

\section{Patient characteristics:}

Of the 1,020 metastatic UC patients available from RISC nomogram database, 52 were excluded due to receipt of $<3$ or $>9$ cycles of chemotherapy and 496 were excluded because they did not fulfill the landmark criterion, leaving 472 patients (receiving cisplatin: $n=338$, or carboplatin: $\mathrm{n}=134$ ) evaluable for the landmark analysis with 281 events (Figure 1 and Table 1$)^{11}$. When examining sites of primary tumors, 381 patients had bladder primary, 87 had upper tract primary, and 4 had unknown sites of primaries; when examining by region of the world, 337 patients were from Europe, 129 patients were from the US and 6 were from Israel. The median follow-up (estimated by the reverse Kaplan-Meier method) of the evaluable population was 35.8 months (IQR: 23.1-55.8). A total of 157 pts received 3 to 5 cycles (median 4) and 315 received 6 to 9 cycles (median 6). The median OS of the evaluable population was 21.8 months and the age and gender characteristics were typical for this population (Table 1). When comparing the group that received 3-5 cycles versus 6-9 cycles, there were no statistically-significant differences for type of platinum, ECOG-PS, leukocytosis, BMI and ethnicity; however, visceral metastases factor was more frequent in the 6-9 cycle group and prior perioperative chemotherapy was more frequent in the 3-5 cycle group.

\section{Cox model analysis for association of number of platinum cycles and survival:}

There was no significant difference between 3-5 and 6-9 cycles of platinum-based chemotherapy (Hazard Ratio [HR]: 1.02, 95\% confidence interval [CI]: 0.78-1.33, p=0.91, Figure 2A, Table 2). Similarly, there were no significant differences between exactly 4 and 6 cycles (HR: 0.89, 95\% CI: 0.60-1.33, $\mathrm{p}=0.57$, Figure 2B), and between $3-5$ vs. 6 vs. $7-9$ cycles (overall p-value 0.877 , Table 2 ).

No significant interactions were observed with type of platinum (Figure $2 \mathrm{C}, \mathrm{p}=0.09$ ) and "completed planned chemotherapy" indication $(\mathrm{p}=0.56)$. Similar findings were obtained after excluding those with progressive disease as the best response to platinum-based chemotherapy and who were not excluded by landmark analysis ( $\mathrm{n}=448$ selected patients): 
HR for $6-9$ versus $3-5$ cycles was 1.03 (95\%CI: $0.78-1.37, \mathrm{p}=0.81$ ). No differential association was observed with survival for $3-5$ versus $6-9$ cycles when examining by RISC nomogram-defined prognostic risk group tertiles (Figure 3A-C).

\section{Discussion}

While advanced UC trials have targeted the delivery of 6 cycles of platinum-based chemotherapy, this is based on arbitrary decisions and randomized trials have not been designed to inform the optimal number of cycles. In recent meta-analyses that were conducted on trials for cisplatin-eligible or ineligible patients, the median number of cycles administered in each study was 4 (range 3-6) for cisplatin-gemcitabine, 4.5 (range 4-6) for taxane, cisplatin, gemcitabine studies and 5.5 (range 4-7) for gemcitabine-taxane studies and 4 (3-6) for gemcitabine-carboplatin studies ${ }^{12,13}$. Moreover, platinum-based combination chemotherapy is characterized by multiple toxicities and decline in performance status, which may undermine the ability to institute second-line therapy and negatively impact on quality of life and survival. Additionally, given the ongoing clinical investigations of second-line switch maintenance therapy using PD-1 or PD-L1 inhibitors in those with stable of responding disease after first-line therapy, the feasibility of this approach will be optimized if patients are not burdened by residual toxicities from first-line therapy. Indeed, ongoing second-line switch maintenance trials require at least 4 cycles of prior platinum-based chemotherapy and not 6 cycles (, ). Moreover, given the potential addition of other new agents (e.g. enfortumab vedotin) and combinations (e.g. docetaxel plus ramucirumab) to the post-platinum therapeutic armamentarium, the absence of lingering overlapping toxicities of first-line platinum-based chemotherapy, especially neuropathy, will facilitate the delivery of a larger number of lines of agents sequentially ${ }^{14,15}$. In this context, it is noteworthy that the optimal duration of PD-1 and PD-L1 inhibitors in the first-line cisplatin-ineligible and post-platinum settings also remains unresolved and should be examined $^{6,7,16-21}$. It is reasonable to hypothesize that optimization of the duration of PD-1 and PD-L1 inhibitors will facilitate the delivery of subsequent third-line therapy.

This retrospective analysis of the large individual patient level RISC database with excellent follow-up (median 35.8 months) demonstrates that 3-5 cycles of platinum based first-line combination chemotherapy may be adequate to treat advanced UC. Patients with early removal from therapy with $<3$ cycles due to toxicities or progression (or other reasons) had to be excluded to avoid confounding, which leads to a relatively chemosensitive population for the analysis (reflected by the somewhat favorable median survival of approximately 22 months for the entire cohort). The clinical characteristics of patients were quite balanced for the $<6$ versus $\geq 6$ cycles groups, with no consistently unfavorable features in any specific group (Table 1). There was no significant difference in OS between those receiving 3-5 versus 6-9 cycles of platinum-based first-line chemotherapy (HR 1.02, $\mathrm{p}=0.91$ ). We also observed no significant interactions with the type of platinum and "completed planned chemotherapy" as defined by the treating physician. Secondary analyses including comparison of exactly 4 versus exactly 6 cycles and 3-5 versus 6 versus 7-9 cycles also yielded no significant differences for association with OS. When examining the data for impact of number of cycles of first-line platinum-based combinations across malignancies, the results observed generally do not suggest that a large number of cycles may yield 
significant increments. Metastatic small cell and non-small cell lung cancer have been treated with 4 cycles since data do not support a benefit for a larger number of cycles ${ }^{22-24}$. In the case of good risk metastatic germ cell tumors, 4 cycles of cisplatin-based combination chemotherapy (bleomycin, etoposide, cisplatin) were not proven to be superior to 3 cycles $^{25,26}$. Although patients with intermediate- or poor risk germ cell tumors continue to receive 4 cycles of BEP, there are no data to support the use of more than 4 cycles, and cumulative toxicity is feared. In the case of metastatic colorectal cancer, oxaliplatin-based chemotherapy is conventionally interrupted after $3-4$ cycles to avoid cumulative neurotoxicity, and recent data also suggest that 3 months of adjuvant therapy may be adequate $^{27-29}$. In the case of ovarian cancer, 6 cycles of platinum-based chemotherapy are targeted by convention, with no data refining the necessity of 6 cycles ${ }^{30}$.

One may hypothesize that patients in the good prognostic group may benefit to a greater extent when employing more than 6 cycles owing to more favorable chemosensitive disease and better tolerance of adverse effects. We did exclude those receiving $>9$ cycles in order to avoid a bias generated by including such patients who are likely to be enriched for outlying patients with extremely durable benefit. However, no differential association was observed with survival for 3-5 versus 6-9 cycles when examining by nomogram-defined risk group tertiles. We did exclude those who received less than 3 cycles for any reason to reduce confounding for early removal for unaccountable reasons since removal for PD, toxicities or patient decision are all possible. We also conducted a supportive sub-analysis removing those with the best response of PD at any time point (for the few who remained evaluable after applying landmark analysis) and this did not show a difference for 3-5 versus 6-9 cycles. While different cutoffs instead of $<3$ and $>9$ cycles could have been used to exclude patients, these criteria may be arbitrary but appear reasonable.

Limitations of a hypothesis-generating retrospective analysis apply. We cannot exclude a benefit in a minor subset of patients who receive 6 or more cycles. In this context, the low prognostic risk group tertile appeared to exhibit a separation of survival with $\searrow 6$ cycles, but this did not attain statistical significance (Figure 3A). A sub-analysis of patients who had at least a stable disease after 6 cycles may have been complementary, but these data were unavailable since radiographic assessments were not performed at identical periodic intervals in all patients and although best response was captured, the response category was not captured at the time of every radiographic examination. The correlation of toxicities with number of cycles would also be a valuable complementary analysis. Patients who tolerate the therapy better may receive a larger number of cycles, and consequently, demonstrate better outcomes. Indeed, it is intriguing that despite this confounding factor that would bias the analysis in favor of a larger number of cycles, we could not identify such an association. Conversely, some toxicities are a clinical pharmacodynamic marker for the activity of therapy and may be associated with better outcomes. While we excluded patients removed for any reason who received less than 3 cycles, we did not have capture of toxicities beyond $\geq 3$ cycles in this dataset to perform an analysis of association of toxicities (nephrotoxicity, neurotoxicity) with number of cycles and survival. 


\section{Conclusions}

Four cycles of platinum based first-line combination chemotherapy may be adequate to treat advanced UC. The practice of administering 6 cycles requires scrutiny since fewer cycles will avert toxicities and facilitate better transition to second-line and switch maintenance therapy strategies being evaluated in trials. The ongoing phase III FOCUS trial being conducted by the South Korean Study Group () is comparing 4 versus 6 cycles of cisplatinbased chemotherapy to better inform clinical decision making.

\section{Supplementary Material}

Refer to Web version on PubMed Central for supplementary material.

\section{Funding:}

This work was supported in part by National Cancer Institute Cancer Center Support grant P30 CA008748.

\section{Glossary}

$\begin{array}{ll}\text { UC } & \begin{array}{l}\text { urothelial carcinoma } \\ \text { RISC }\end{array} \\ \text { Retrospective International Study of Invasive/Advanced Cancer of } \\ \text { the Urothelium } \\ \text { OS } & \text { programmed death-1 } \\ \text { PD-1 } & \text { programmed death-ligand-1 } \\ \text { PD-L1 } & \text { Eastern Cooperative Oncology Group performance status }\end{array}$

\section{References}

1. von der Maase H, Sengelov L, Roberts JT et al.: Long-term survival results of a randomized trial comparing gemcitabine plus cisplatin, with methotrexate, vinblastine, doxorubicin, plus cisplatin in patients with bladder cancer. J Clin Oncol, 23: 4602, 2005 [PubMed: 16034041]

2. Sternberg CN, de Mulder P, Schornagel JH et al.: Seven year update of an EORTC phase III trial of high-dose intensity M-VAC chemotherapy and G-CSF versus classic M-VAC in advanced urothelial tract tumours. Eur J Cancer, 42: 50, 2006 [PubMed: 16330205]

3. Saxman SB, Propert KJ, Einhorn LH et al.: Long-term follow-up of a phase III intergroup study of cisplatin alone or in combination with methotrexate, vinblastine, and doxorubicin in patients with metastatic urothelial carcinoma: a cooperative group study. J Clin Oncol, 15: 2564, 1997 [PubMed: 9215826]

4. Bellmunt J, von der Maase H, Mead GM et al.: Randomized phase III study comparing paclitaxel/ cisplatin/gemcitabine and gemcitabine/cisplatin in patients with locally advanced or metastatic urothelial cancer without prior systemic therapy: EORTC Intergroup Study 30987. J Clin Oncol, 30: 1107, 2012 [PubMed: 22370319]

5. De Santis M, Bellmunt J, Mead G et al.: Randomized phase II/III trial assessing gemcitabine/ carboplatin and methotrexate/carboplatin/vinblastine in patients with advanced urothelial cancer who are unfit for cisplatin-based chemotherapy: EORTC study 30986. J Clin Oncol, 30: 191, 2012 [PubMed: 22162575] 
6. Balar AV, Galsky MD, Rosenberg JE et al.: Atezolizumab as first-line treatment in cisplatinineligible patients with locally advanced and metastatic urothelial carcinoma: a single-arm, multicentre, phase 2 trial. Lancet, 389: 67, 2017 [PubMed: 27939400]

7. Balar AV, Castellano D, O'Donnell PH et al.: First-line pembrolizumab in cisplatin-ineligible patients with locally advanced and unresectable or metastatic urothelial cancer (KEYNOTE-052): a multicentre, single-arm, phase 2 study. Lancet Oncol, 2017

8. Galsky MD, Hahn NM, Rosenberg J et al.: Treatment of patients with metastatic urothelial cancer "unfit" for Cisplatin-based chemotherapy. J Clin Oncol, 29: 2432, 2011 [PubMed: 21555688]

9. Chovanec M, Abu Zaid M, Hanna N et al.: Long-term toxicity of cisplatin in germ-cell tumor survivors. Ann Oncol, 28: 2670, 2017 [PubMed: 29045502]

10. Seng S, Liu Z, Chiu SK et al.: Risk of venous thromboembolism in patients with cancer treated with Cisplatin: a systematic review and meta-analysis. J Clin Oncol, 30: 4416, 2012 [PubMed: 23150697]

11. Necchi A, Sonpavde G, Lo Vullo S et al.: Nomogram-based Prediction of Overall Survival in Patients with Metastatic Urothelial Carcinoma Receiving First-line Platinum-based Chemotherapy: Retrospective International Study of Invasive/Advanced Cancer of the Urothelium (RISC). Eur Urol, 71: 281, 2017 [PubMed: 27726966]

12. Giannatempo P, Pond GR, Sonpavde G et al.: The Impact of Adding Taxanes to Gemcitabine and Platinum Chemotherapy for the First-Line Therapy of Advanced or Metastatic Urothelial Cancer: A Systematic Review and Meta-analysis. Eur Urol, 69: 624, 2016 [PubMed: 26497923]

13. Necchi A, Pond GR, Raggi D et al.: Efficacy and Safety of Gemcitabine Plus Either Taxane or Carboplatin in the First-Line Setting of Metastatic Urothelial Carcinoma: A Systematic Review and Meta-Analysis. Clin Genitourin Cancer, 15: 23, 2017 [PubMed: 27324051]

14. Petrylak DP, P R, Zhang J, et al.: A Phase I Study of Enfortumab Vedotin: Updated Analysis of Patients with Metastatic Urothelial Cancer. J Clin Oncol 35, 2017 (suppl; abstr 106)

15. Petrylak DP, de Wit R, Chi KN et al.: Ramucirumab plus docetaxel versus placebo plus docetaxel in patients with locally advanced or metastatic urothelial carcinoma after platinum-based therapy (RANGE): a randomised, double-blind, phase 3 trial. Lancet, 2017

16. Rosenberg JE, Hoffman-Censits J, Powles T et al.: Atezolizumab in patients with locally advanced and metastatic urothelial carcinoma who have progressed following treatment with platinum-based chemotherapy: a single-arm, multicentre, phase 2 trial. Lancet, 387: 1909, 2016 [PubMed: 26952546]

17. Sharma P, Retz M, Siefker-Radtke A et al.: Nivolumab in metastatic urothelial carcinoma after platinum therapy (CheckMate 275): a multicentre, single-arm, phase 2 trial. Lancet Oncol, 2017

18. Powles T, O'Donnell PH, Massard C et al.: Efficacy and Safety of Durvalumab in Locally Advanced or Metastatic Urothelial Carcinoma: Updated Results From a Phase 1/2 Open-label Study. JAMA Oncol: e172411, 2017 [PubMed: 28817753]

19. Bellmunt J, de Wit R, Vaughn DJ et al.: Pembrolizumab as Second-Line Therapy for Advanced Urothelial Carcinoma. N Engl J Med, 2017

20. Patel MR, Ellerton J, Infante JR et al.: Avelumab in metastatic urothelial carcinoma after platinum failure (JAVELIN Solid Tumor): pooled results from two expansion cohorts of an open-label, phase 1 trial. Lancet Oncol, 19: 51, 2018 [PubMed: 29217288]

21. Sonpavde G: PD-1 and PD-L1 Inhibitors as Salvage Therapy for Urothelial Carcinoma. N Engl J Med, 376: 1073, 2017 [PubMed: 28212061]

22. Patel JD, Socinski MA, Garon EB et al.: PointBreak: a randomized phase III study of pemetrexed plus carboplatin and bevacizumab followed by maintenance pemetrexed and bevacizumab versus paclitaxel plus carboplatin and bevacizumab followed by maintenance bevacizumab in patients with stage IIIB or IV nonsquamous non-small-cell lung cancer. J Clin Oncol, 31: 4349, 2013 [PubMed: 24145346]

23. Schiller JH, Harrington D, Belani CP et al.: Comparison of four chemotherapy regimens for advanced non-small-cell lung cancer. N Engl J Med, 346: 92, 2002 [PubMed: 11784875]

24. Roth BJ, Johnson DH, Einhorn LH et al.: Randomized study of cyclophosphamide, doxorubicin, and vincristine versus etoposide and cisplatin versus alternation of these two regimens in extensive 
small-cell lung cancer: a phase III trial of the Southeastern Cancer Study Group. J Clin Oncol, 10: 282, 1992 [PubMed: 1310103]

25. Einhorn LH, Williams SD, Loehrer PJ et al.: Evaluation of optimal duration of chemotherapy in favorable-prognosis disseminated germ cell tumors: a Southeastern Cancer Study Group protocol. J Clin Oncol, 7: 387, 1989 [PubMed: 2465391]

26. Saxman SB, Finch D, Gonin R et al.: Long-term follow-up of a phase III study of three versus four cycles of bleomycin, etoposide, and cisplatin in favorable-prognosis germ-cell tumors: the Indian University experience. J Clin Oncol, 16: 702, 1998 [PubMed: 9469360]

27. Shi Q, Sobrero AF, Shields AF et al.: Prospective pooled analysis of six phase III trials investigating duration of adjuvant (adjuv) oxaliplatin-based therapy (3 vs 6 months) for patients (pts) with stage III colon cancer (CC): The IDEA (International Duration Evaluation of Adjuvant chemotherapy) collaboration. Journal of Clinical Oncology, 35: LBA1, 2017

28. de Gramont A, Buyse M, Abrahantes JC et al.: Reintroduction of oxaliplatin is associated with improved survival in advanced colorectal cancer. J Clin Oncol, 25: 3224, 2007 [PubMed: 17664470]

29. Chibaudel B, Maindrault-Goebel F, Lledo G et al.: Can chemotherapy be discontinued in unresectable metastatic colorectal cancer? The GERCOR OPTIMOX2 Study. J Clin Oncol, 27: 5727, 2009 [PubMed: 19786657]

30. Oza AM, Cook AD, Pfisterer J et al.: Standard chemotherapy with or without bevacizumab for women with newly diagnosed ovarian cancer (ICON7): overall survival results of a phase 3 randomised trial. Lancet Oncol, 16: 928, 2015 [PubMed: 26115797] 
$\mathrm{N}=1,020$ who received first-line platinum-based chemotherapy

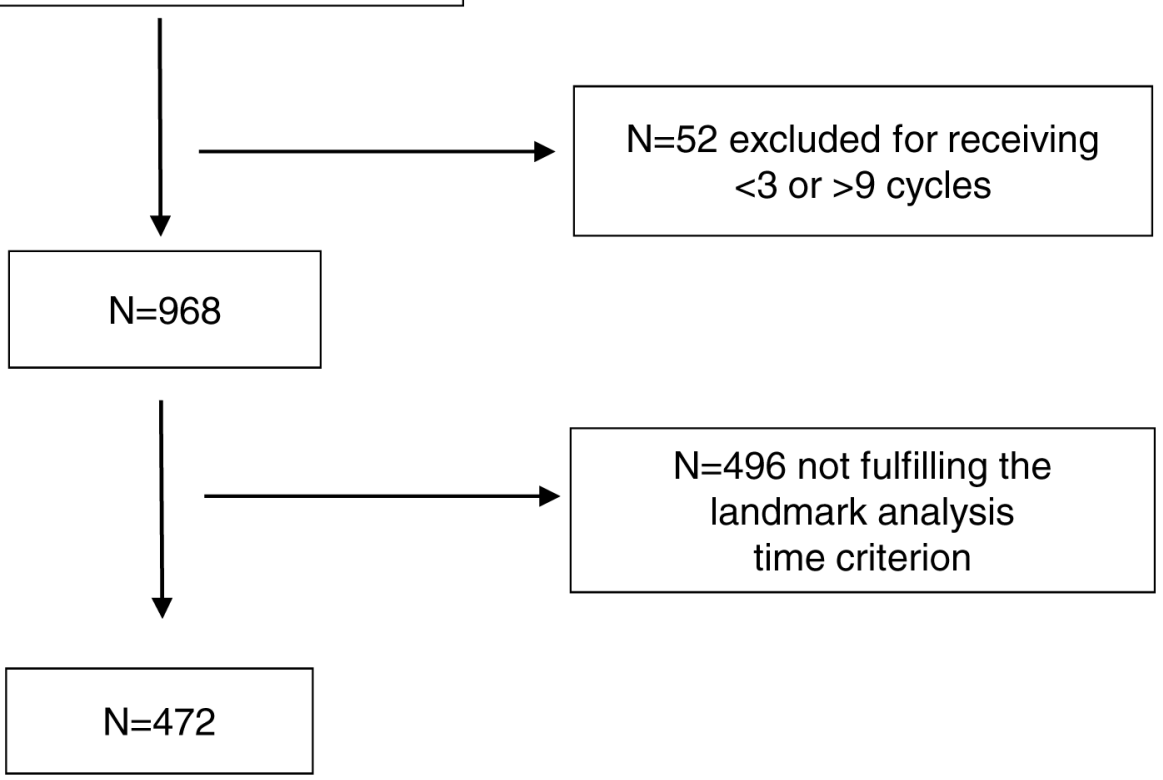

Figure 1. Patient selection flow chart none 


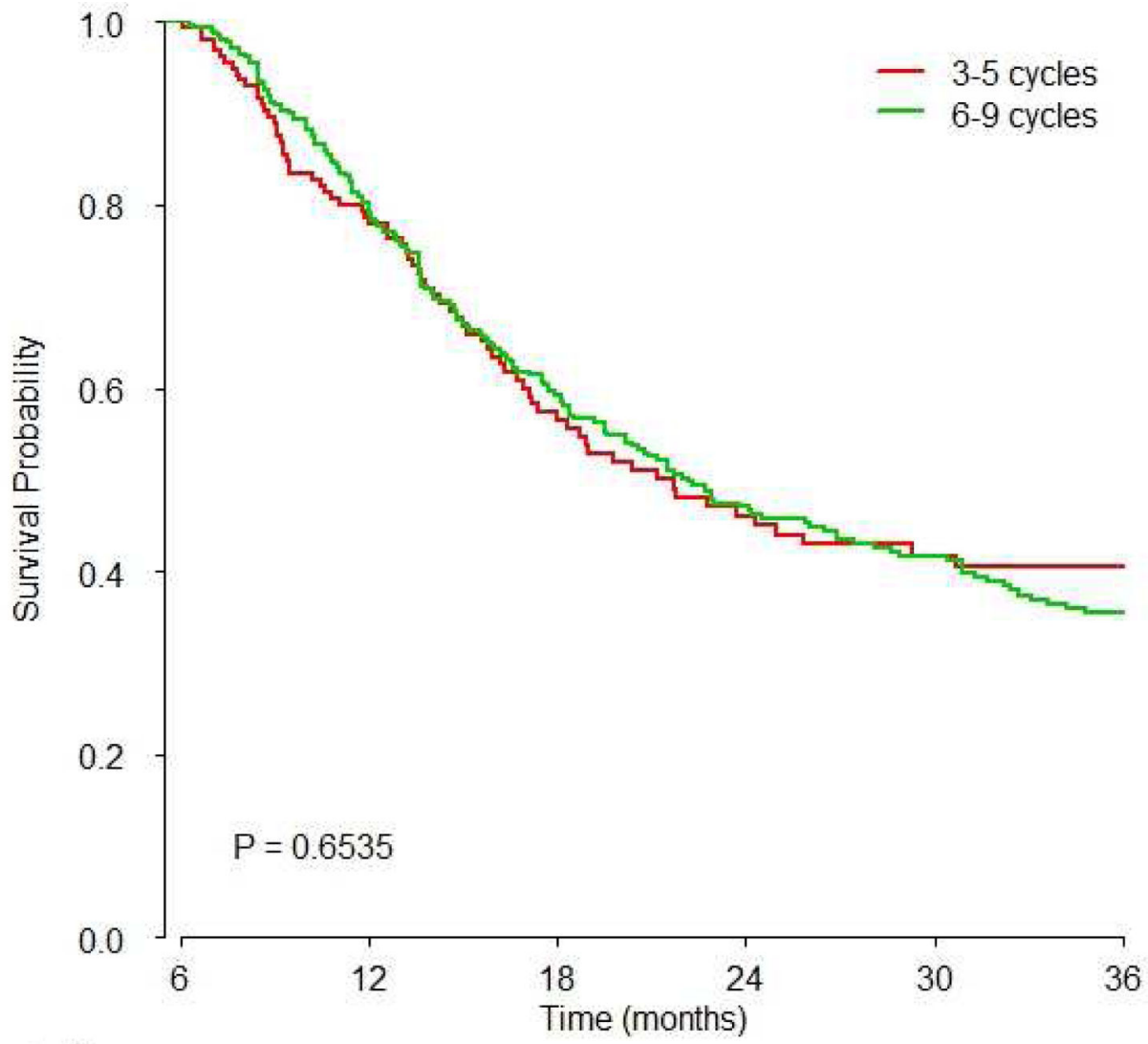

No. pts at risk

$\begin{array}{rrrrrrr}3-5 \text { cycles } & 157 & 111 & 64 & 44 & 34 & 22 \\ 6-9 \text { cycles } & 315 & 234 & 161 & 114 & 90 & 64\end{array}$




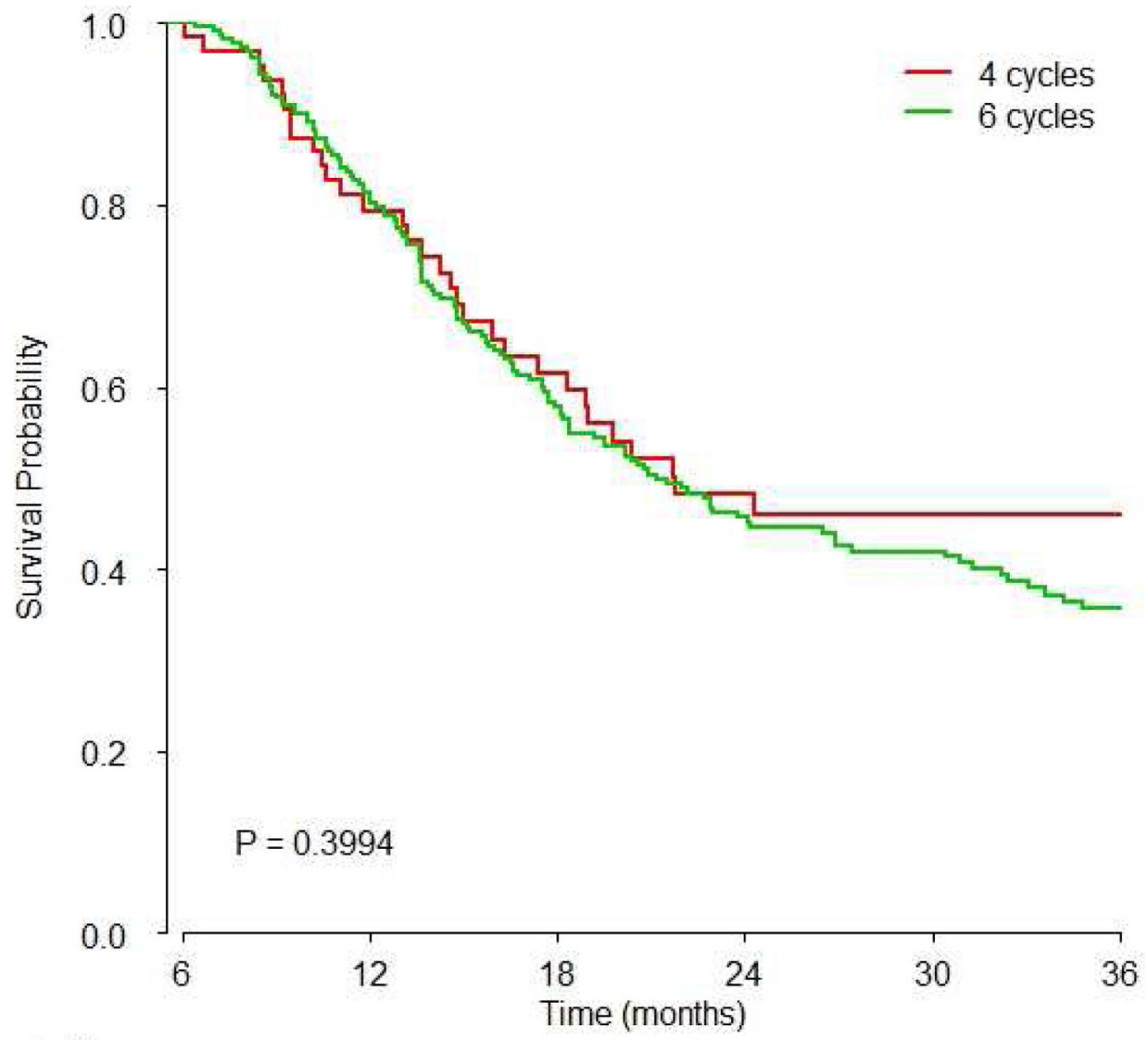

No. pts at risk

$\begin{array}{lrrrrrr}4 \text { cycles } & 65 & 49 & 33 & 22 & 19 & 12 \\ 6 \text { cycles } & 236 & 180 & 118 & 81 & 64 & 46\end{array}$




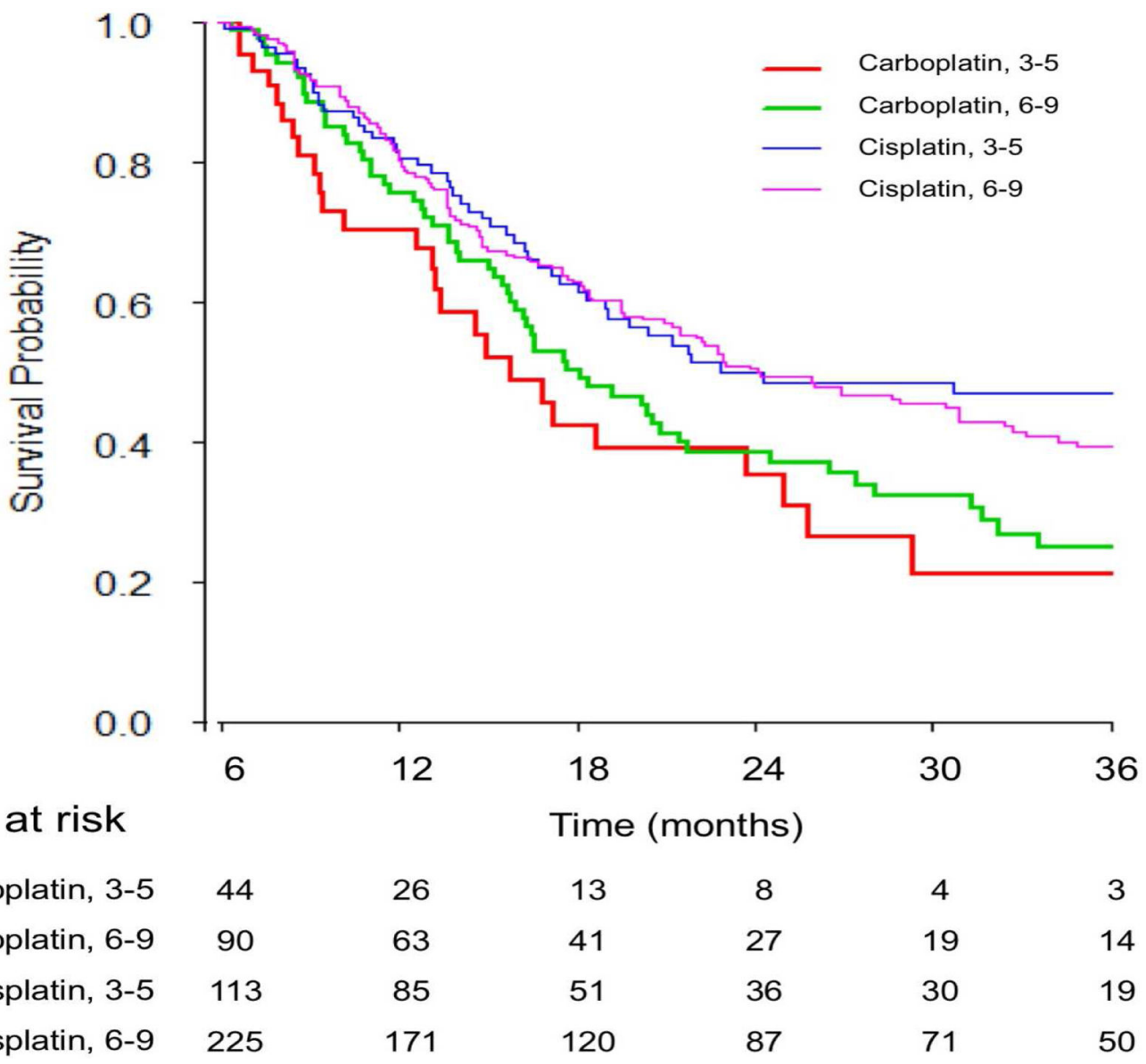

Figure 2. Survival based on A) 3-5 vs. 6-9 cycles overall, B) 4 vs. 6 cycles overall and C) 3-5 vs. 6-9 cycles based on platinum agent

Kaplan-Meier overall survival curves are shown and $p$ values were obtained by the logrank test showing:

A) There was no significant difference of survival between 3-5 vs. 6-9 cycles of chemotherapy (HR 1.02, 95\% CI: 0.77-1.33, $\mathrm{p}=0.9$ );

B) Comparison of exactly 4 vs. exactly 6 cycles showed no significant difference in survival (HR: 0.89, 95\%CI: 0.60-1.33, p=0.39)

C) No significant difference of survival was observed between 3-5 vs. 6-9 cycles of carboplatin or cisplatin-based chemotherapy 


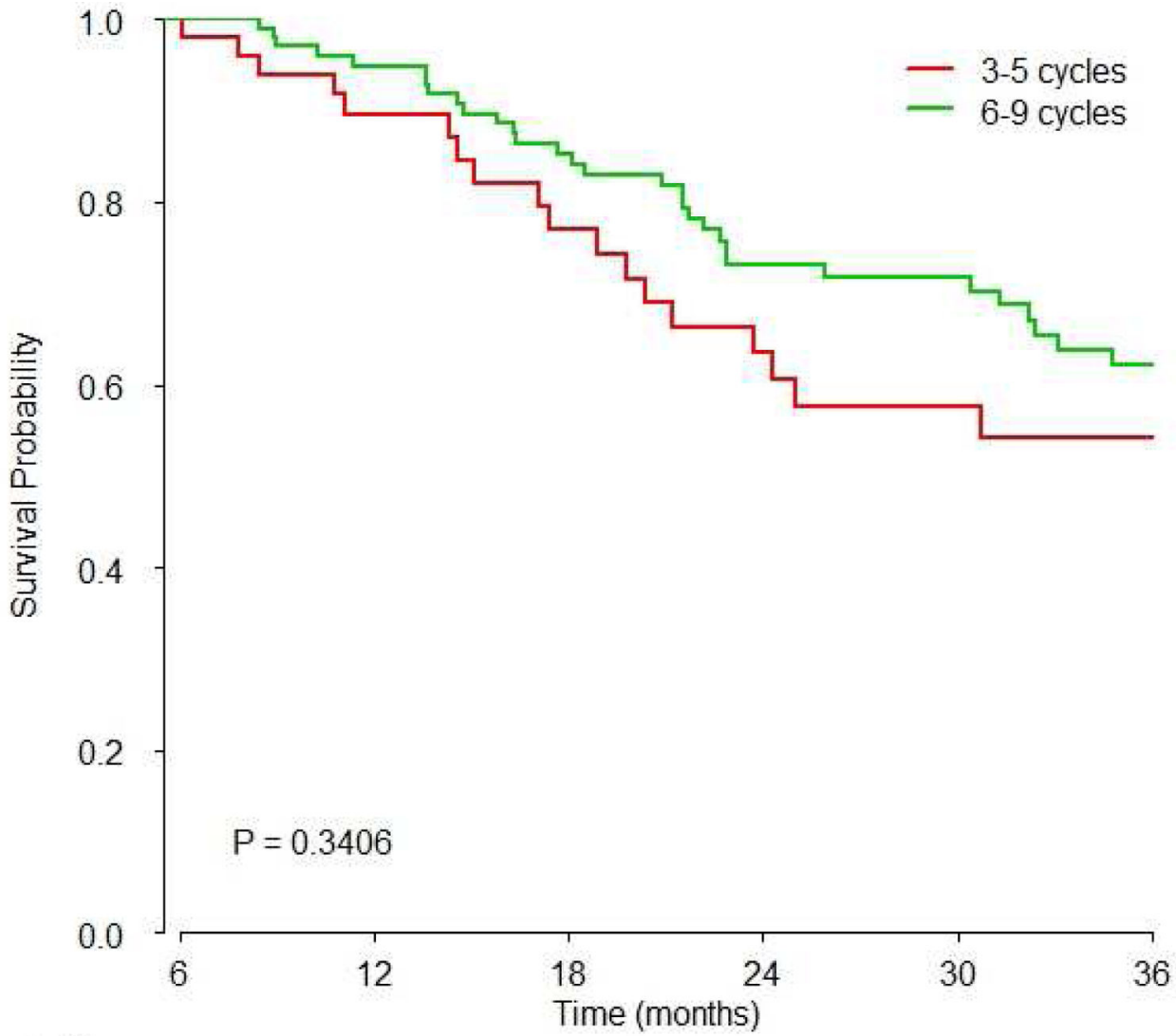

No. pts at risk

3-5 cycles

$6-9$ cycles
41

90
29

74
22

56
18

48
11

33 


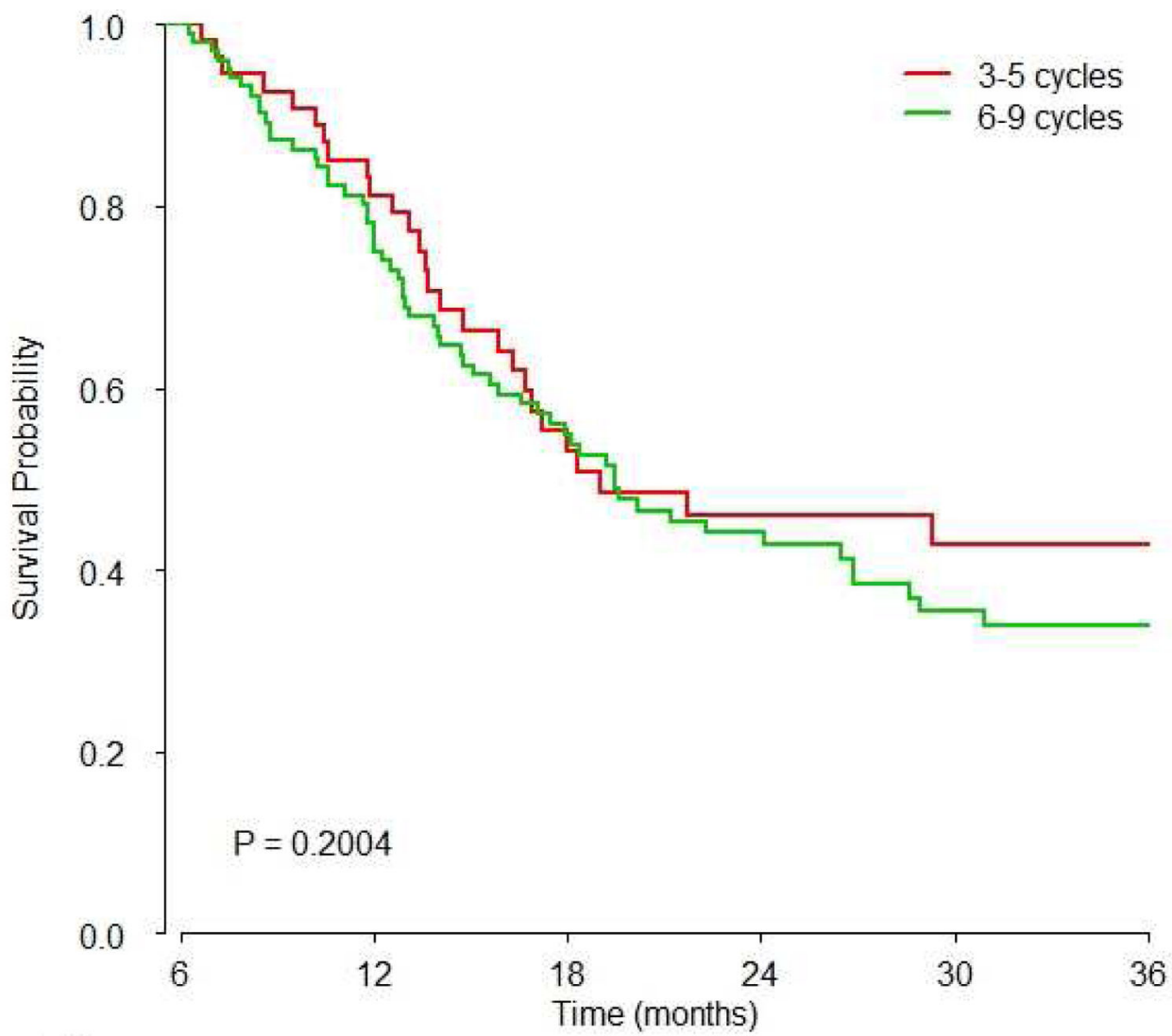

No. pts at risk

$\begin{array}{rrrrrrr}3-5 \text { cycles } & 55 & 42 & 25 & 16 & 13 & 8 \\ 6-9 \text { cycles } & 102 & 76 & 48 & 33 & 23 & 20\end{array}$




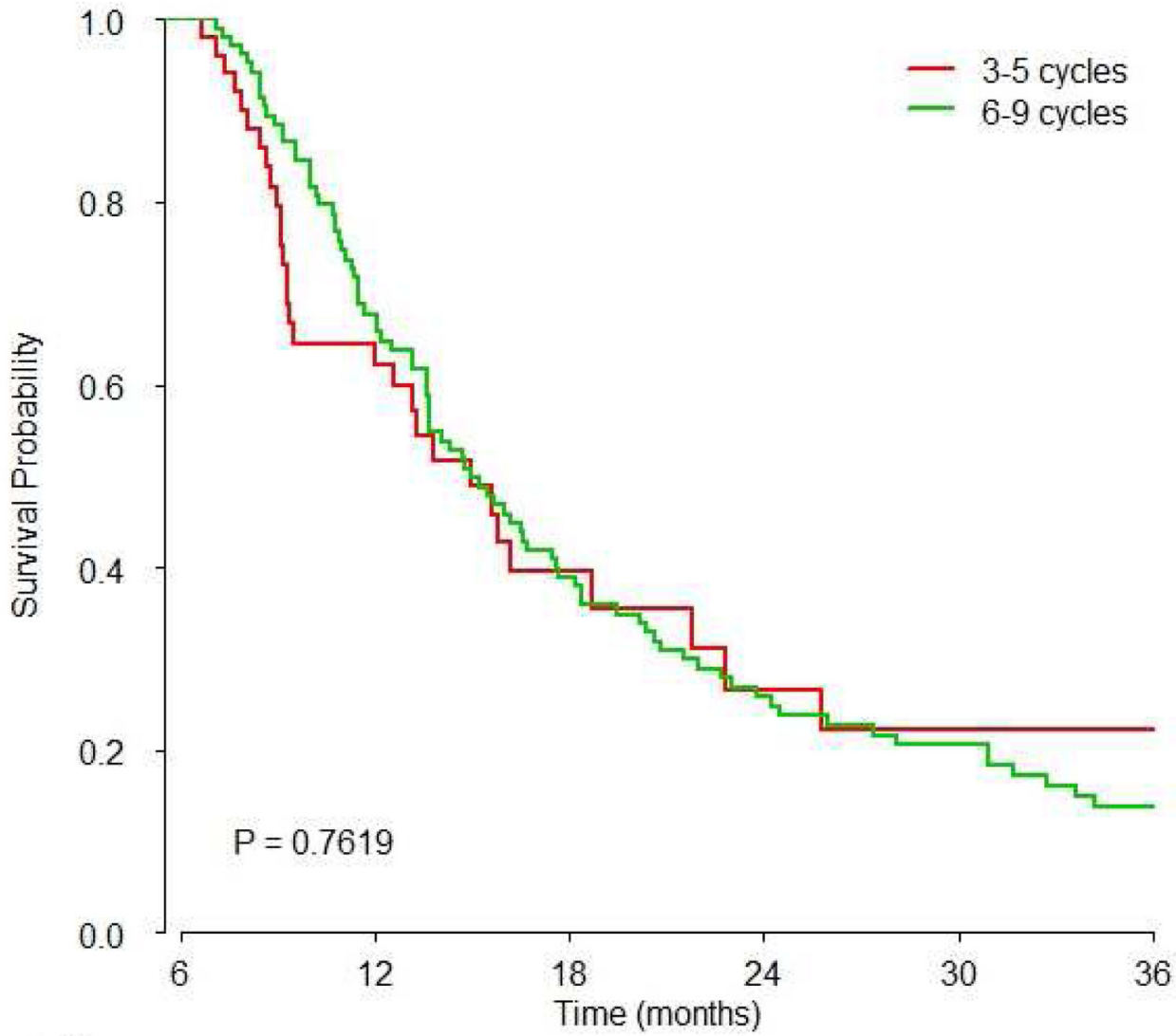

No. pts at risk

$\begin{array}{rrrrrrr}3-5 \text { cycles } & 51 & 28 & 10 & 6 & 3 & 3 \\ 6-9 \text { cycles } & 106 & 68 & 39 & 25 & 19 & 11\end{array}$

Figure 3. Survival based on prognostic risk group

Kaplan-Meier overall survival curves are shown and $\mathrm{p}$ values were obtained by the logrank test showing no differential association with survival for 3-5 vs. 6-9 cycles when examining defined risk group tertiles based on the previously published prognostic nomogram for survival (A: low risk, B: intermediate risk, $\mathrm{C}$ : high risk) ${ }^{11}$ 


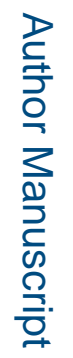

\begin{tabular}{|c|c|c|c|c|c|c|c|c|c|c|c|c|c|c|c|c|c|c|c|c|c|c|}
\hline & 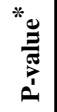 & 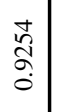 & & 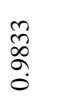 & & & 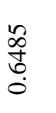 & & $\begin{array}{l}\overline{0} \\
\vdots \\
0\end{array}$ & $\stackrel{\circ}{\stackrel{0}{0}}$ & & $\mid \begin{array}{l}0 \\
\stackrel{0}{0} \\
\stackrel{0}{0}\end{array}$ & & $\frac{\widetilde{m}}{\stackrel{2}{0}}$ & & & $\begin{array}{l}\frac{\infty}{3} \\
\stackrel{0}{0}\end{array}$ & 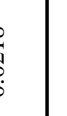 & $\begin{array}{l}\frac{T}{\Delta} \\
\stackrel{\alpha}{\circ}\end{array}$ & & 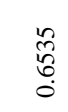 & \\
\hline $\begin{array}{l}z \\
y \\
\frac{w}{0}\end{array}$ & $\therefore$ & $\begin{array}{l}\widehat{T} \\
\hat{D} \\
0 \\
0\end{array}$ & $\begin{array}{c}\underset{?}{\square} \\
\infty \\
\infty\end{array}$ & $\begin{array}{l}\hat{\infty} \\
\stackrel{\infty}{=}\end{array}$ & & 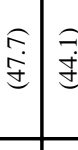 & $\underset{\infty}{\curvearrowright}$ & & $\begin{array}{l}0 \\
0 \\
0 \\
\dot{f} \\
0 \\
0\end{array} \mid$ & $\begin{array}{l}6 \\
\stackrel{+}{ \pm} \\
\dot{ \pm}\end{array}$ & 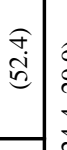 & $\begin{array}{l}\hat{\sigma} \\
\vec{i} \\
\dot{j} \\
\dot{J}\end{array}$ & 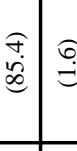 & $\begin{array}{l}\sigma \\
\dot{c}\end{array}$ & $\stackrel{o}{\oplus}$ & $\underset{f}{\stackrel{f}{+}}$ & $\stackrel{\widehat{\infty}}{\stackrel{\oplus}{\oplus}}$ & 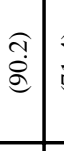 & $\stackrel{\Im}{\stackrel{\Im}{S}}$ & 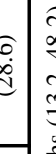 & 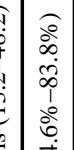 & $\begin{array}{l}0 \\
0 \\
0 \\
n \\
n \\
1 \\
0 \\
n\end{array}$ \\
\hline e & $\mathrm{z}$ & 6 & ฉٌ & in & - & \begin{tabular}{l|l} 
\pm & \pm \\
\end{tabular} & $\pi$ & 吕 & ㄱ. & $\stackrel{n}{n}$ & $\stackrel{n}{6}$ & & 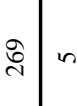 & 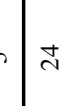 & $m$ & \pm & $\bar{m}$ & 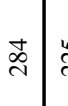 & તે & 2 & 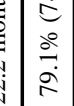 & 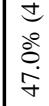 \\
\hline
\end{tabular}



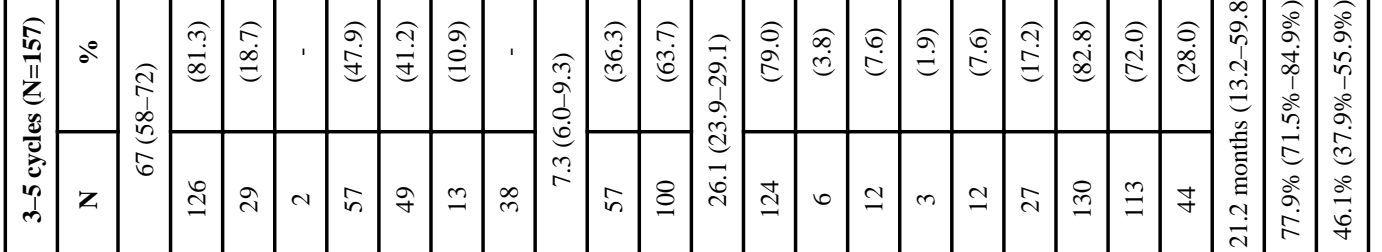

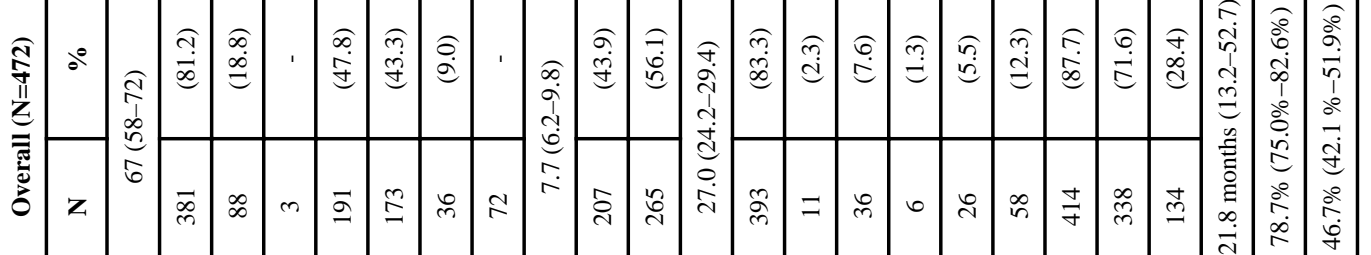

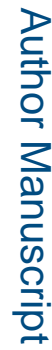

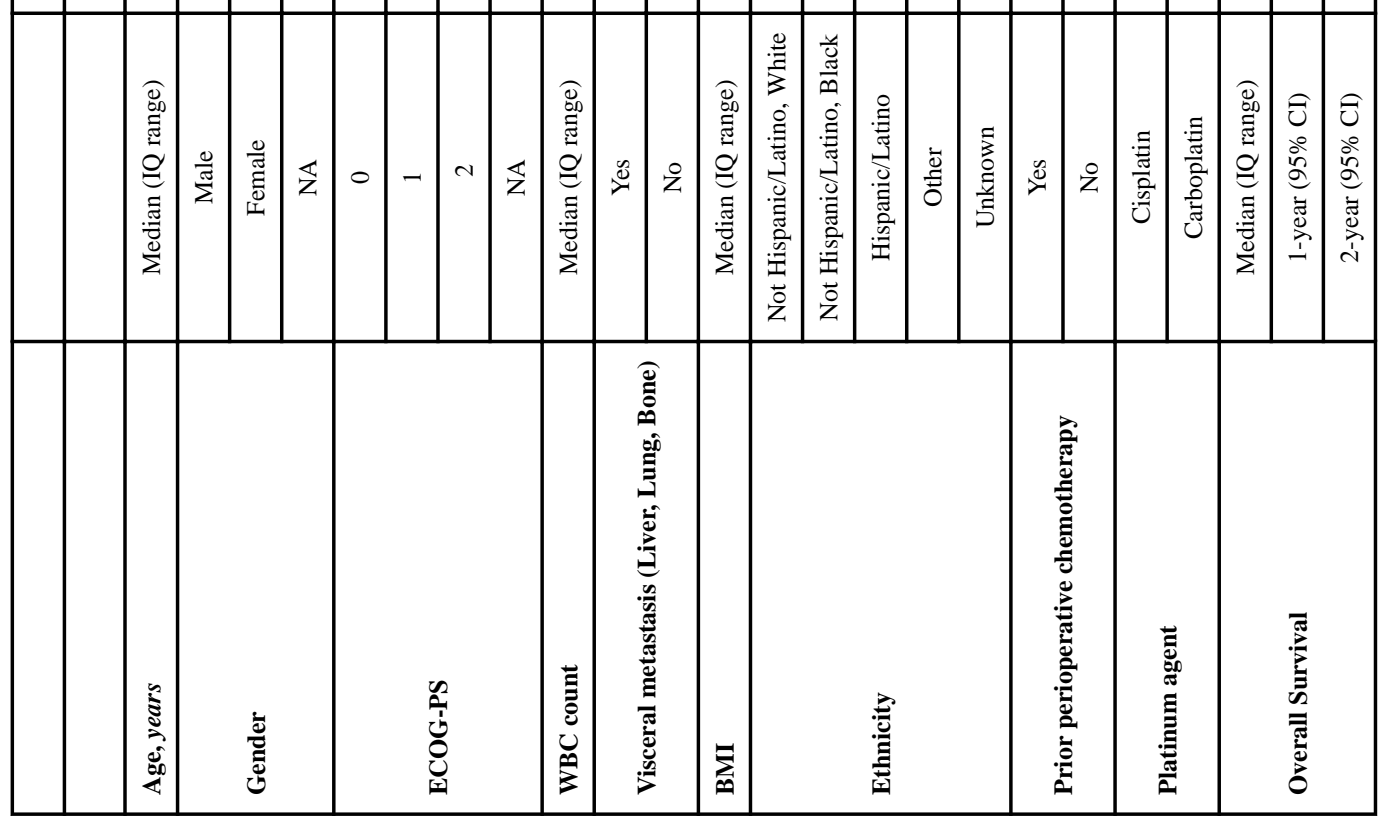

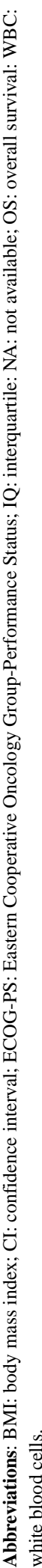



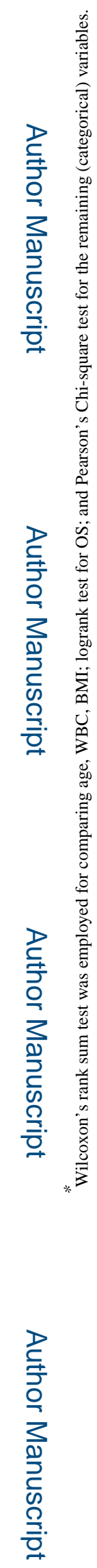

J Urol. Author manuscript; available in PMC 2019 December 01. 


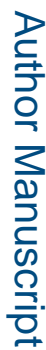

ก่
$\frac{0}{0}$
$\frac{0}{\pi}$

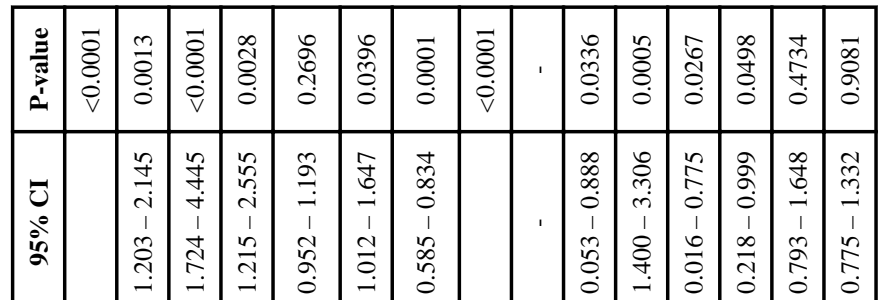

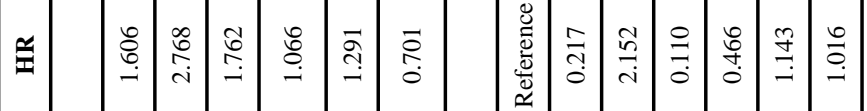

\begin{tabular}{|c|c|c|c|c|c|c|c|c|c|c|c|}
\hline 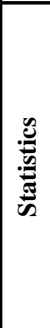 & $\begin{array}{l}0 \\
\vdots \\
2\end{array}$ & $\begin{array}{l}0 \\
\dot{1} \\
\vec{\lambda}\end{array}$ & $\begin{array}{l}0 \\
\dot{n} \\
\bar{z} \\
z\end{array}$ & 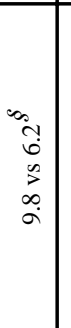 & 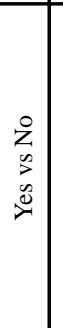 & 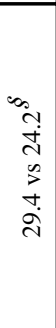 & 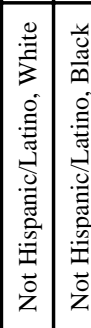 & 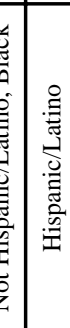 & 它 & $\begin{array}{l}\text { है } \\
\text { है } \\
\text { है }\end{array}$ & 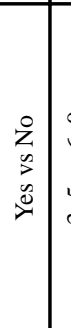 \\
\hline 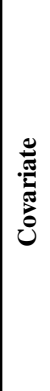 & 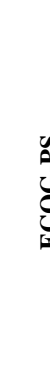 & 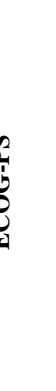 & & 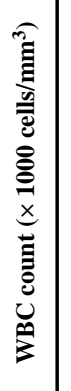 & 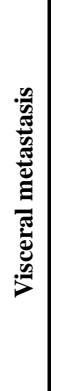 & $\sum_{\infty}^{E}$ & & 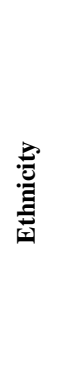 & & & 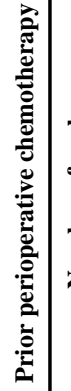 \\
\hline
\end{tabular}

